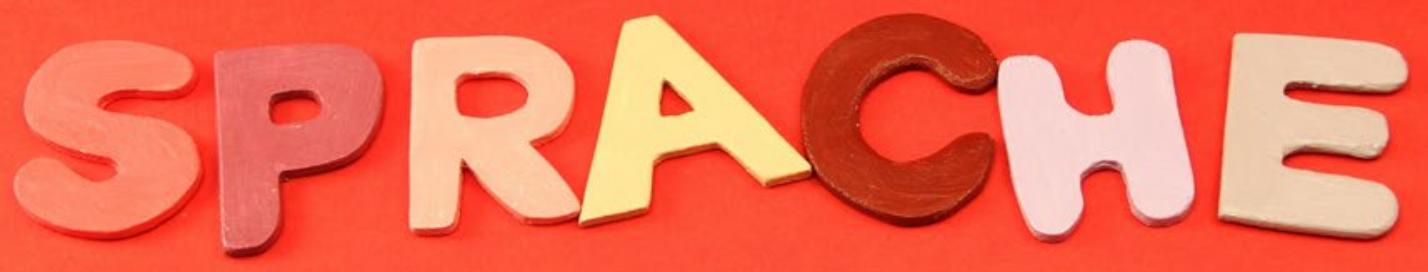

\title{
Sprachliche und religiöse Hürden bei der Diabetestherapie von Migranten
}

\begin{abstract}
Menschen mit Diabetes mellitus, die aus einem anderen Kultur- bzw. Sprachraum stammen, sind für den behandelnden Arzt eine Herausforderung. Es gilt, die Besonderheiten im Krankheitsverständnis, bei der Lebensführung, der Ernährung und der Compliance zu berücksichtigen. Denn auch Traditionen, Sitten, Bräuche und Tabus können Auswirkungen auf die Stoffwechselkontrolle haben.
\end{abstract}

Diabetespatienten mit einem Migrationshintergrund werden in Deutschland immer häufiger. In der Mehrzahl der Fälle handelt es sich heute noch um Typ 1-Diabetiker. Aber je länger Migranten in Deutschland leben, umso mehr werden sie sich an die westlichen Ernährungs- und Lebensbedingungen anpassen mit der Folge, dass auch vermehrt ein Typ-2-Diabetes manifest wird. Die Betreuung und Behandlung solcher Patienten ist mit einer Reihe von besonderen Problemen verbunden, so Sr. Innocentia Chisara Omumuh, Krankenschwester und Pflegepädagogin aus Mainz. Das Hauptproblem bei diabetischen Migranten sind die

\footnotetext{
Schulungsmaterial der DDG und KBV zum Download

Mit den Aspekten und Fragen der Kultursensibilität innerhalb der patientenorientierten und interdisziplinären Versorgung von Patienten mit Diabetes mellitus befasst sich etwa die Arbeitsgemeinschaft (AG) Diabetes und Migranten der Deutschen Diabetes-Gesellschaft (DDG).

Sie ist eine offene Arbeitsgemeinschaft, sodass sich neben den DDGMitgliedern auch interessierte Nichtmitglieder einbringen können. Die Projektgruppen widmen sich einzelnen Themen im Kontext „Diabetes und Migranten" und werden nach Bedarf aktiv beauftragt. Die Arbeitsgemeinschaft trifft ihre verbindlichen Entscheidungen normalerweise auf den jährlichen Mitgliederversammlungen. Diese finden regelmäßig im Rahmen der DDG-Frühjahrskongresse und bei Bedarf auch im Rahmen der DDG-Herbsttagungen statt. Die Arbeitsgemeinschaft ist auch kontinuierliche Teilnehmerin des Diabetes Forums, das jährlich bei der DDG-Herbsttagung stattfindet. Dort werden die Aktivitäten der AG Diabetes und Migranten vorgestellt.
}

Hier gibt es fremdsprachliches Schulungsmaterial der DDG kostenlos zum Download: http://migration.deutsche-diabetes-gesellschaft.de/ arbeitsmaterialien.html

Und auch die Kassenärztliche Bundesvereinigung stellt entsprechendes Material zur Verfügung: www.kbv.de/html/1150_20914.php unzureichenden Sprachkenntnisse. Deshalb sind für die Beratung und Schulung fremdsprachliche Arbeitsmaterialien unverzichtbar. Sehr hilfreich sind Behandlungsbilderbücher mit gut erfassbaren Illustrationen. Soweit machbar ist es sinnvoll, sprachkundige Angehörige einzubinden. Stehen solche nicht zur Verfügung, muss ein Dolmetscher hinzugezogen werden. Diese seien jedoch emotional nicht so engagiert wie die Angehörigen, die deshalb immer den Vorzug haben sollten. Wichtig ist auch, dass der Patient zu einer Diabetesberaterin ein Vertrauensverhältnis aufbauen kann. Besonders vorteilhaft ist es, wenn eine der Diabetesberaterinnen selbst einen Migrationshintergrund hat. Grundsätzlich sollte man solchen Patienten mit viel Verständnis begegnen und sie nicht zu schnell als schulungsunfähig abqualifizieren.

\section{Aufklärung mit den richtigen Mitteln}

Gerade bei Patientinnen mit einem Schwangerschaftsdiabetes spielen auch religiöse Aspekte eine wichtige Rolle. Ist eine Insulintherapie erforderlich, so sie zum Teil abgelehnt, weil fälschlicherweise angenommen wird, dass es sich bei dem zu spritzenden Insulin um Schweineinsulin handelt. Dann kann es sinnvoll sein, einen muslimischen religiösen Führer einzubeziehen. Dies gilt auch im Hinblick auf die Einhaltung des Fastenmonats Ramadan. 80 \% aller muslimischen Patienten mit einem Typ2-Diabetes wollen nämlich während des Ramadan fasten. Dabei stellen sich eine Reihe von Fragen: Muss die Therapie umgestellt werden? Kommt es zu Hypo- oder Hyperglykämien oder auch zu einer Gewichtszunahme? Bei Patienten, die bisher mit einem Sulfonylharnstoff behandelt werden, kann die Umstellung auf ein Therapieprinzip ohne Hypoglykämierisiko wie Gliptin oder GLP-1-Analogon durchaus sinnvoll sein.

Dr. med. Peter Stiefelhagen

Quelle: Herbsttagung der Deutschen Diabetesgesellschaft, 11.11.2016 in Nürnberg 